\title{
Pollen Quantity and Viability in 65 Litchi (Litchi chinensis Sonn.) Cultivars
}

\author{
Jiefang W $\mathbf{u}^{1}$, Danwen Fu $\mathbf{u}^{1}$, Jiezhen Chen, Changhe Cai, Qian Yan, \\ and Liangxi $\mathrm{Ou}^{2}$
}

Institute of Fruit Tree Research, Guangdong Academy of Agricultural Sciences, Guangzhou City 510640, Guangdong, China; and Key Laboratory of South Subtropical Fruit Biology and Genetic Resource Utilization, Ministry of Agriculture, Guangzhou City 510640, Guangdong, China

Additional index words. litchi germplasm resources, pollen amount, pollen germination rate, cluster analysis, male flower stage

\begin{abstract}
The characteristics of litchi pollen have drawn increasing attention in recent years. Previous studies indicated that there are significant differences in the quantity and viability of litchi pollen grains among different varieties and flowering stages. Moreover, the same variety may show a different quantity of pollen grains and viability in different years. There is still a lack of systematic studies on the change of pollen germination rate and pollen amount in different varieties and at different flowering stages. In this study, the changes in the germination rate of pollen at different development stages were studied. It was primarily revealed that the pollen germination rate already approached its peak upon the filament extends fully, but the anther does not dehisce the developmental stage. In 2009 and 2010, the viability and number of pollen grains per anther were investigated in 65 litchi cultivars, and a difference was observed among cultivars. The pollen germination rate ranged between $20.14 \%$ ('Wuchali') and $54.69 \%$ ('Donlongmili'). The number of pollen grains per anther ranged between 1555 ('Zhongshanzhuangyuanhong') and 7455 ('Houye'). Sixty-five litchi cultivars were classified into six clusters based on the pollen quantity and germination rate. Most litchi cultivars can produce large amounts of viable and compatible pollen grains during the flowering period. Thus, our results indicate that the pollen amount and germination rate might not be the only factors restricting the successful pollination of litchi.
\end{abstract}

Litchi (Litchi chinensis Sonn.) originated in southern China and northern Vietnam but has now spread to countries with subtropical climates (Menzel and Waite, 2005). Litchi is not only an important fruit in China, India, Vietnam, and Thailand but is also cultivated in Australia, South Africa, the United States, and South America. A major factor affecting the production of this subtropical fruit in many countries is its low yield ( $\mathrm{Yu}$ et al., 2015). The trees may fail to flower some seasons or fail to set and carry a reasonable crop (Menzel, 2001). Aizen and Harder (2007) considered pollen limitation as the combined effect of quantity and quality limitation, indicating that quantity limitation is less frequent than was inferred from pollen supplementation experiments. Moreover, their results suggested that an expanded broad perspective that recognized

Received for publication 8 June 2017. Accepted for publication 21 Aug. 2017.

This study was supported by the National Litchi \& Longan Industry Technology System-Germplasm Resources Evaluation Posts (CAR-33-01), National Infrastructure for Crop Germplasm Resources (NICGR 2016-53), Crop Germplasm Resources Protection (1120162130135252017), and the Litchi Germplasm Resources Protection (16RZZY-05).

${ }^{1}$ These authors contributed equally to this work. ${ }^{2}$ Corresponding author. E-mail: gdglxo2015@126. com. the fecundity consequences of pollination with poor-quality pollen would improve the ecological understanding of pollen limitation.

The litchi flower is a type of unisexual flower for which self-pollination is not common (Xiang et al., 2001). Litchi is a gynaecandrous and monoecious plant, yet gynaecandrous pollination or geitonogamy does not commonly occur because male and female flowers do not appear simultaneously. Moreover, litchi is a typical cross-pollinated plant; the productive cultivars (except very few varieties or individual plants with natural parthenocarpic ability) do not bear fruit unless they are pollinated and fertilized. However, the viability of pollen usually affects pollination, fertilization, and fruit set rate, so it is necessary to study the characteristics of litchi pollen for cultivation and breeding.

For entomophilous flowers, the amount of pollen grains deposited on the stigma depends on the number of pollinator visits received by a floret during a certain period (visit rate) and the proportion of viable pollen in the pollen loads carried by the pollinators (Dafni et al., 2005). Litchi is a typical out-pollinated plant, and fruit set can occur only if pollen from male flowers is transferred to the stigma of the female flowers (Stern and Gazit, 1996). Insufficient pollination is an important factor responsible for low yields in litchi. Litchi pollination requires large amounts of pollen grains during the flowering period. However, not much information is available about the relative levels of the quality and number of pollen grains among different litchi cultivars. Moreover, a better understanding of the relationship between the number and quality of pollen grains among cultivars may help in choosing cultivars for litchi orchards to improve the fruit set.

The study concerning the characteristics of litchi pollen has drawn increasing attention in recent years. Previous studies have indicated that there is a great difference in pollen viability among varieties (Ou et al., 2010). Moreover, the same variety may show distinctly different pollen viabilities in different years; flowering may be advanced or delayed significantly by weather at the development stage of anthers in the same year (Xiang et al., 1994), and flowering may also be affected by air temperature at the stage of germination (Xu et al., 1982). Litchi pollen germination rate and pollen amount have been reported in many studies (Jiang et al., 2012; Ou et al., 2010; Stern and Gazit 1998; Xiang et al., 2000); however, systematic studies on the change of pollen germination rate and pollen amount from variety to variety at different flowering stages are still lacking. In this study, the changes in the germination rate of pollen at different development stages were studied. In 2009 and 2010 , the viability and number of pollen grains per anther were investigated in 65 litchi cultivars. More precise information is needed in several major areas, including the selection of a parent, the collection of pollen, and the determination of hybridization time in the crossbreeding of litchi. The objective of this study was to evaluate the number of pollen grains per anther and the pollen germination capacity of different cultivars of litchi trees.

\section{Materials and Methods}

Plant material. The field experiments were conducted in the years 2009 and 2010 at the Litchi Germplasm Repository, Guangzhou, China. Guangzhou belongs to the subtropical monsoon climate region. The wet season was mainly concentrated from April to June. In 2009 and 2010, litchi flowers bloom mainly during March to April in Guangzhou. The average temperature was $17.45^{\circ} \mathrm{C}$ during March to April in 2009. The average temperature was $19.75^{\circ} \mathrm{C}$ during March to April in 2010. The rainy season started on 28 Mar. 2009. The rainy season started on $22 \mathrm{Apr}$. 2010. Guangzhou belongs to a hilly area and the altitude is from 200 to $500 \mathrm{~m}$. Sixty-nine litchi cultivars were used as test plants (Tables 1 and 2). The 25- to 30-year-old trees of these pollen parents were healthy and free from diseases and pests.

Pollen collection and counting. Fifty anthers were collected from the filament extends of fully male flowers during the second wave of blooming. The anthers were placed in 2-mL centrifuge tubes and then dried at 
Table 1. The difference of the pollen germination in litchi male flower types and stages.

\begin{tabular}{|c|c|c|c|c|c|c|c|c|}
\hline \multirow[b]{2}{*}{ Stages } & \multicolumn{8}{|c|}{ Pollen germination $(\%)$} \\
\hline & $\begin{array}{l}\text { Sanyuehong } \\
(\mathrm{M} 1)^{\mathrm{z}}\end{array}$ & $\begin{array}{l}\text { Sanyuehong } \\
\text { (M2) }\end{array}$ & $\begin{array}{l}\text { Shuidong } \\
\text { (M1) }\end{array}$ & $\begin{array}{l}\text { Shuidong } \\
\text { (M2) }\end{array}$ & $\begin{array}{c}\text { Zhuangyuanhong } \\
\text { (M1) }\end{array}$ & $\begin{array}{l}\text { Zhuangyuanhong } \\
\text { (M2) }\end{array}$ & $\begin{array}{c}\text { Songxiang } \\
\text { (M1) }\end{array}$ & $\begin{array}{l}\text { Songxiang } \\
\text { (M2) }\end{array}$ \\
\hline First stage & $2.3 \pm 0.95 \mathrm{~cd}$ & $7.8 \pm 0.13 \mathrm{~d}$ & $0.65 \pm 0.04 \mathrm{c}$ & $3.62 \pm 0.085 \mathrm{~d}$ & $0.71 \pm 0.02 \mathrm{c}$ & $3.72 \pm 0.04 \mathrm{~cd}$ & $2.42 \pm 0.05 \mathrm{~d}$ & $12.65 \pm 0.17 \mathrm{~d}$ \\
\hline Second stage & $16.9 \pm 1.01 \mathrm{~b}$ & $28.3 \pm 1.65 b$ & $1.74 \pm 0.056 \mathrm{~b}$ & $7.27 \pm 0.2 \mathrm{c}$ & $1.71 \pm 0.036 \mathrm{~b}$ & $6.26 \pm 0.11 \mathrm{c}$ & $7.54 \pm 0.18 \mathrm{c}$ & $24.76 \pm 0.22 b$ \\
\hline Third stage & $22.5 \pm 1.32 \mathrm{a}$ & $43.35 \pm 1.26 \mathrm{a}$ & $1.96 \pm 0.02 \mathrm{a}$ & $10.0 \pm 0.5 \mathrm{a}$ & $1.99 \pm 0.05 \mathrm{a}$ & $9.28 \pm 0.1 \mathrm{a}$ & $10.0 \pm 0.4 \mathrm{a}$ & $28.92 \pm 0.66 \mathrm{a}$ \\
\hline Fourth stage & $4.40 \pm 0.2 \mathrm{c}$ & $21.5 \pm 0.5 \mathrm{c}$ & $0.30 \pm 0.01 \mathrm{~d}$ & $9.27 \pm 0.06 \mathrm{~b}$ & $0.20 \pm 0.01 \mathrm{~d}$ & $9.13 \pm 0.03 \mathrm{~b}$ & $8.56 \pm 0.05 \mathrm{~b}$ & $17.96 \pm 0.1 \mathrm{c}$ \\
\hline
\end{tabular}

${ }^{\mathrm{z}}$ Different letters indicate significant difference among stages at $P=0.05$ (Tukey's test).

$35^{\circ} \mathrm{C}$ with an air-blowing electric dryer for $24 \mathrm{~h}$ until the anthers were completely dry. The tubes were vibrated to release the pollen from the anthers. About $2 \mathrm{~mL}$ of distilled water was added to each tube. The centrifuge tube was then placed in an ultrasonic oscillator for $15 \mathrm{~min}$ and vortexed for $5 \mathrm{~min}$ to evenly suspend the pollen in water. Pollen quantity was calculated by the blood count method. Briefly, a drop of the suspension was placed in a hemocyte counting plate (XB-K25 ) to count the number of pollen under a microscope. The number of pollen in a large square $(1.0 \times 1.0 \times 0.1 \mathrm{~mm}$ in size with 400 small squares) was counted. Each treatment was performed in triplicates. The quantity of pollen in one anther was computed as the quantity of pollen/one anther $=\left(2 \times n / 10^{-4}\right) / 50$; where $n$ represents the mean number of pollen in the large square, 2 represents $2 \mathrm{~mL}$ of suspension, and $10^{-4}$ represents the volume of the large square, and 50 represents the number of anthers.

Pollen germination. The litchi has three types of flowers: Male 1 (M1), Male 2 (M2), and Female $(\mathrm{F})$. The three types coming into anthesis in three distinct waves: the first is the male (M1) bloom, the second is the female bloom, and the third is the male bloom (M2). Male flower development of litchi was defined in four stages (Fig. 1). In the first stage, the anther extended just beyond the receptacle, and the filaments were not observed. In the second stage, the filament is extended to half of its overall length. In the third stage, the filament extends fully, but the anther does not dehisce, and in the last stage, the filament extends fully, and the anther dehisces. Anthers were collected from each of the four stages during the first and second wave of blooming for 'Sanyuehong', 'Shuidong', 'Zhuangyuanhong', and 'Songxiang'. Anthers were collected from the third stage during the second wave of blooming for the 65 identification varieties. Pollen grains were collected, dehydrated, and scattered on $1 \%(\mathrm{w} / \mathrm{v})$ solid agar, which was supplemented with $3 \%$ sucrose $(w / v)$ in petri dishes. The growth of the pollen tubes was observed under a light microscope after incubation at $30{ }^{\circ} \mathrm{C}$ for $3 \mathrm{~h}$ (Jiang et al., 2012). Pollen germination was observed under a microscope $(40 \times)$. Twenty different fields of vision, each with at least 30 grains, were examined per treatment. Pollen grains were considered to have germinated when the pollen tube was at least as long as the diameter of the pollen grain.
Statistical analysis. Data obtained were analyzed by analysis of variance (ANOVA). The percentages of viable pollen that were below $30 \%$ or over $70 \%$ were transformed to the Arcsin P 0.5 for ANOVA analysis. The statistical significance of the treatments was tested using Tukey's test at 5\% probability. Statistical analyses were performed using the SPSS 19.0 statistical software. The hierarchical cluster was constructed by the Ward Method clustering using the squared Euclidean distance as the distance metric. The pollen quantity and germination rate of different litchi cultivars were analyzed as variables in hierarchical cluster.

\section{Results}

The difference in pollen germination in different types of litchi male flowers and stages. The development of the male flower can be divided into four stages (Fig. 1). The germination rates of the M1 and M2 in five accessions of germplasm at different developmental stages were tested in this experiment. There was a significant difference in pollen germination rate among the different development stages (Table 1). The germination rate of pollen in the M1 was far below that in the M2, and at the third development stage of male flowers in both types, the male flowers, called filaments, extended fully, and the germination rate of pollen reached its maximum before anther dehiscence. Thus, the anthers in the M2 at the third stage were selected as the research subject in this study.

Pollen quantity and germination rate of different litchi cultivars. Sixty-five litchi cultivars were used as test plants to determine the pollen quantity in 2009 and 2010. A difference was observed among cultivars. The number of pollen grains per anther ranged between 1555 ('Zhongshanzhuangyuanhong') and 7455 ('Houye') (Table 2). The number of pollen grains varied greater than 2000 among the 2 years in 15 cultivars. The cultivars included 'Shuijingqiu', 'Zengchenggualv', 'Changhong', 'Cuixiangli', 'Yuanduanli', 'Liuli 1', 'Liuli 2', 'Huangpili', 'Yanzhihong', 'Dongguanshangshuhuai', 'Cuiye', 'Yiqiaomili', 'Baisheng', 'Wuchali', and 'Haiken 14', and the quantity values were higher in the 2010 year with respect to the 2009 year in 13 varieties.

The pollen germination rate varied among the cultivars. The pollen germination rate of the 65 litchi cultivars ranged between $20.14 \%$ ('Wuchali') and 54.69\% ('Donlongmili') (Table 2). The average number of pollen grains per anther for the 65 litchi cultivars was 4216.22 . The average pollen germination rate of the 65 litchi cultivars was $33.94 \%$. The number of pollen grains varied (greater than 20\%) among the 2 years in nine cultivars, including 'Changwen', 'Jicuirou', 'Zhuanyuan 2', 'Nandaowuheli', 'Qingyuan', 'Xiangwan', 'Yuanduanli', 'Haiken 13', and 'Panguzi'. Moreover, the viability values were higher in 2010 compared with 2009 in five varieties.

Classification of litchi cultivars based on pollen quantity and pollen germination rate. These cultivars could be classified into six clusters at a distance of three (Table 3 ). A significant difference was observed between clusters. The first cluster contained six cultivars with the highest pollen quality. The number of pollen grains per anther ranged between 4935 and 7455, and the pollen germination rate ranged between $45.40 \%$ and $54.69 \%$ in the first cluster. The second cluster contained six cultivars, and the number of pollen grains per anther ranged between 5570 and 6495, and the pollen germination rate ranged between $24.36 \%$ and $32.43 \%$. The third group cluster contained 17 cultivars, the number of pollen grains per anther ranged between 3895 and 5155 , and the pollen germination rate ranged between $29.60 \%$ and $38.15 \%$. The fourth cluster contained 12 cultivars, and the pollen quality was the lowest. The number of pollen grains per anther ranged between 3522 and 4690 , and the pollen germination rate ranged between $20.14 \%$ and $28.51 \%$ in the fourth cluster. The fifth cluster contained 13 cultivars; the number of pollen grains per anther ranged between 2815 and 4472.5, and the pollen germination rate ranged between $35.65 \%$ and $50.30 \%$. The sixth cluster contained 11 cultivars with the lowest pollen quantity. The number of pollen grains per anther ranged between 1555 and 3420, and the pollen germination rate ranged between $21.69 \%$ and $33.10 \%$ in the sixth cluster.

\section{Discussions}

The novel aspects of this research were as follows: first, the changes in the germination rate of pollen at different development stages were studied, revealing that the pollen germination rate already approached its peak on the start of the second development stage; second, the largest number of litchi cultivars were investigated in this study.

Pollen does not develop synchronously in the same anther in litchi. When the male 


\begin{tabular}{|c|c|c|c|c|c|}
\hline \multirow[b]{2}{*}{ Code } & \multirow[b]{2}{*}{ Cultivar name } & \multicolumn{2}{|c|}{ Pollen grains/anther (n.) } & \multicolumn{2}{|c|}{ Pollen germination rate } \\
\hline & & 2009 & 2010 & 2009 & 2010 \\
\hline$\overline{\mathrm{C} 1}$ & Shuijingqiu & $8,080 \pm 102.85$ & $4,910 \pm 82.34$ & $22.65 \pm 0.95$ & $39.66 \pm 1.24$ \\
\hline $\mathrm{C} 2$ & Baipili & $6,400 \pm 133.33$ & $6,660 \pm 68.41$ & $40 \pm 1.31$ & $58.87 \pm 1.64$ \\
\hline $\mathrm{C} 3$ & Zengchenggualv & $6,200 \pm 84.33$ & $1,570 \pm 13.42$ & $24.1 \pm 0.89$ & $39.21 \pm 1.07$ \\
\hline $\mathrm{C} 4$ & Changwen & $5,560 \pm 82.19$ & $6,440 \pm 46.04$ & $17.85 \pm 1.13$ & $37.96 \pm 0.74$ \\
\hline C5 & Jingxing & $5,480 \pm 134.66$ & $4,340 \pm 93.17$ & $34.67 \pm 1.5$ & $24.53 \pm 1.24$ \\
\hline C6 & Hongli & $5,200 \pm 133.33$ & $5,110 \pm 95.50$ & $34.67 \pm 1.43$ & $37.04 \pm 1.2$ \\
\hline $\mathrm{C} 7$ & Guiwei & $5,240 \pm 131.99$ & $5,045 \pm 53.71$ & $35 \pm 0.99$ & $37.21 \pm 1.3$ \\
\hline $\mathrm{C} 8$ & Nuomici & $5,200 \pm 84.33$ & $6,080 \pm 72.94$ & $50.70 \pm 1.69$ & $51.35 \pm 1.41$ \\
\hline C9 & Jicuirou & $4,840 \pm 99.33$ & $4,105 \pm 39.31$ & $29.73 \pm 1.31$ & $56.25 \pm 1.81$ \\
\hline $\mathrm{C} 10$ & Yeshenglizhi 10 & $4,800 \pm 103.3$ & $3,900 \pm 73.42$ & $46.79 \pm 1.33$ & $46 \pm 1.25$ \\
\hline $\mathrm{C} 11$ & Linqingxiaodingxiang & $5,160 \pm 54$ & $3,770 \pm 65.27$ & $21.15 \pm 0.54$ & $28.8 \pm 0.77$ \\
\hline $\mathrm{C} 12$ & Zhuanyuan 1 & $3,240 \pm 60.37$ & $3,040 \pm 66.03$ & $20.34 \pm 1.3$ & $34.57 \pm 1.89$ \\
\hline $\mathrm{C} 13$ & Zhuanyuan 2 & $3,680 \pm 83.8$ & $4,940 \pm 38.47$ & $17.20 \pm 1.17$ & $44.23 \pm 1.58$ \\
\hline $\mathrm{C} 14$ & Ziniangxi & $4,320 \pm 62.54$ & $3,755 \pm 59.87$ & $26.32 \pm 1.13$ & $20.39 \pm 1.13$ \\
\hline $\mathrm{C} 15$ & Nandaowuheli & $3,600 \pm 73.03$ & $4,195 \pm 37.22$ & $16.92 \pm 0.72$ & $39.55 \pm 1.27$ \\
\hline $\mathrm{C} 16$ & Xinqiumili & $4,200 \pm 88.84$ & $4,485 \pm 47.17$ & $21.62 \pm 1.28$ & $20.83 \pm 1.34$ \\
\hline $\mathrm{C} 17$ & Bianli & $3,960 \pm 54$ & $4,085 \pm 41.05$ & $33.96 \pm 0.87$ & $32.39 \pm 1.19$ \\
\hline $\mathrm{C} 18$ & Heiye & $3,960 \pm 70.55$ & $2,490 \pm 45.61$ & $23.26 \pm 0.94$ & $38.95 \pm 2.72$ \\
\hline C19 & Xuehuaizi & $4,440 \pm 89.94$ & $3,250 \pm 84.26$ & $25.71 \pm 1.59$ & $24.62 \pm 2.74$ \\
\hline $\mathrm{C} 20$ & Xiaoye & $3,560 \pm 82.19$ & $3,000 \pm 53.29$ & $29.38 \pm 1.17$ & $31.15 \pm 1.93$ \\
\hline $\mathrm{C} 21$ & Changhong & $3,360 \pm 56.57$ & $5,800 \pm 70.71$ & $25.49 \pm 1.57$ & $43.04 \pm 1.27$ \\
\hline $\mathrm{C} 22$ & Feizixiao & $3,320 \pm 46.19$ & $3,725 \pm 43.99$ & $14.43 \pm 0.94$ & $28.07 \pm 0.77$ \\
\hline $\mathrm{C} 23$ & Dadingxiang & $4,160 \pm 56.57$ & $5,670 \pm 79.25$ & $23.19 \pm 1.82$ & $38.09 \pm 0.78$ \\
\hline $\mathrm{C} 24$ & Shangshuhuai & $3,080 \pm 50.77$ & $4,280 \pm 66.93$ & $51.4 \pm 1.69$ & $48.62 \pm 1.31$ \\
\hline $\mathrm{C} 25$ & Heimiandeng & $2,960 \pm 52.49$ & $2,640 \pm 47.33$ & $34 \pm 1.49$ & $18.29 \pm 1.02$ \\
\hline $\mathrm{C} 26$ & Qingyuan & $2,880 \pm 46.19$ & $4,390 \pm 74.7$ & $8.72 \pm 0.71$ & $36.44 \pm 1.28$ \\
\hline $\mathrm{C} 27$ & Tongshachihuaizhi & $2,800 \pm 55.78$ & $1,830 \pm 57.10$ & $23.33 \pm 1$ & $22.81 \pm 0.83$ \\
\hline $\mathrm{C} 28$ & Shuidong & $2,120 \pm 35.28$ & $2,420 \pm 30.33$ & $29.41 \pm 1.31$ & $28.77 \pm 1.83$ \\
\hline $\mathrm{C} 29$ & Zhongshanzhuangyuanhong & $1,240 \pm 27.65$ & $1,870 \pm 34.35$ & $30.53 \pm 1.26$ & $24 \pm 0.89$ \\
\hline $\mathrm{C} 30$ & Xiangwan & $5,600 \pm 94.66$ & $5,280 \pm 75.17$ & $57.3 \pm 0.64$ & $34.5 \pm 0.7$ \\
\hline C31 & Cuixiangli & $5,280 \pm 86.72$ & $7,340 \pm 64.5$ & $35.8 \pm 0.4$ & $28.98 \pm 0.83$ \\
\hline $\mathrm{C} 32$ & Donglongmili & $5,160 \pm 78.77$ & $5,000 \pm 76.75$ & $58.4 \pm 1.01$ & $50.97 \pm 1.33$ \\
\hline $\mathrm{C} 33$ & Chunteng & $4,960 \pm 52.15$ & $4,500 \pm 32.25$ & $35.6 \pm 0.67$ & $33.21 \pm 0.88$ \\
\hline $\mathrm{C} 34$ & Houye & $6,910 \pm 85.09$ & $8,000 \pm 68.12$ & $47.2 \pm 0.89$ & $43.6 \pm 0.56$ \\
\hline $\mathrm{C} 35$ & Guangxigoubei & $4,880 \pm 66.27$ & $4,440 \pm 50.99$ & $35.3 \pm 0.78$ & $27.95 \pm 0.88$ \\
\hline $\mathrm{C} 36$ & Yutanmili & $4,700 \pm 70.68$ & $5,170 \pm 23.24$ & $52 \pm 0.7$ & $46.46 \pm 0.79$ \\
\hline C37 & Tianyan & $4,680 \pm 87.02$ & $3,160 \pm 33.47$ & $45.5 \pm 1.17$ & $41 \pm 1.61$ \\
\hline $\mathrm{C} 38$ & Yuanduanli & $4,680 \pm 70.68$ & $6,780 \pm 58.65$ & $35 \pm 1.2$ & $13.72 \pm 0.56$ \\
\hline C39 & Huaizhi & $4,420 \pm 50.95$ & $2,930 \pm 56.04$ & $23.23 \pm 0.47$ & $22.38 \pm 0.61$ \\
\hline $\mathrm{C} 40$ & Hongjun 1 & $4,360 \pm 54.32$ & $4,570 \pm 36.33$ & $26.5 \pm 0.6$ & $28.5 \pm 0.84$ \\
\hline C41 & Liuli 1 & $4,160 \pm 45.22$ & $6,980 \pm 68.12$ & $17.2 \pm 0.66$ & $32.6 \pm 0.77$ \\
\hline $\mathrm{C} 42$ & Liuli 2 & $3,408 \pm 42.21$ & $6,790 \pm 70.99$ & $32.5 \pm 0.72$ & $35 \pm 0.79$ \\
\hline $\mathrm{C} 43$ & Haiken 13 & $4,040 \pm 38.87$ & $3,620 \pm 58.31$ & $53 \pm 1.28$ & $32.1 \pm 0.91$ \\
\hline $\mathrm{C} 44$ & Wumian & $4,032 \pm 47.96$ & $2,080 \pm 40.5$ & $29.3 \pm 0.72$ & $33.5 \pm 0.7$ \\
\hline $\mathrm{C} 45$ & Jiayuanmili & $3,880 \pm 33.73$ & $4,030 \pm 53.85$ & $38.7 \pm 0.78$ & $29.1 \pm 0.86$ \\
\hline $\mathrm{C} 46$ & Anduojidan & $3,840 \pm 38.64$ & $5,000 \pm 68.12$ & $38.3 \pm 0.88$ & $30.1 \pm 0.82$ \\
\hline $\mathrm{C} 47$ & Tianshuili & $3,720 \pm 57.19$ & $1,910 \pm 47.12$ & $42.8 \pm 0.89$ & $41.14 \pm 0.74$ \\
\hline $\mathrm{C} 48$ & Huangpili & $3,600 \pm 46.95$ & $8,460 \pm 41.95$ & $32.6 \pm 0.84$ & $32.25 \pm 0.66$ \\
\hline C49 & Yanzhihong & $3,560 \pm 57.19$ & $5,820 \pm 48.99$ & $20.6 \pm 0.62$ & $31.5 \pm 0.69$ \\
\hline C50 & Aili & $3,520 \pm 50.6$ & $5,350 \pm 50$ & $25 \pm 0.95$ & $39.1 \pm 0.41$ \\
\hline C51 & Leilingxiaodingxiang & $3,440 \pm 65.86$ & $4,960 \pm 50.99$ & $44.2 \pm 0.7$ & $40.49 \pm 0.61$ \\
\hline C52 & Chengtuo & $3,200 \pm 40.88$ & $2,840 \pm 50.99$ & $43.7 \pm 0.73$ & $27.6 \pm 0.65$ \\
\hline C53 & Heizhi & $3,200 \pm 57.97$ & $5,020 \pm 58.31$ & $35.7 \pm 0.91$ & $40.6 \pm 0.67$ \\
\hline C54 & Houxian & $3,200 \pm 15.78$ & $3,640 \pm 24.49$ & $37.2 \pm 0.64$ & $29 \pm 0.94$ \\
\hline C55 & Panguzi & $3,160 \pm 36.27$ & $3,660 \pm 54.41$ & $55 \pm 0.85$ & $21 \pm 0.66$ \\
\hline C56 & Dongguanshangshuhuai & $3,000 \pm 52.99$ & $5,390 \pm 76.55$ & $38.5 \pm 0.62$ & $34.5 \pm 0.72$ \\
\hline C57 & Mupai 1 & $3,000 \pm 57.81$ & $4,920 \pm 86.02$ & $43.1 \pm 0.69$ & $42.25 \pm 0.74$ \\
\hline C58 & Cuiye & $2,880 \pm 50.42$ & $4,910 \pm 40$ & $37.1 \pm 0.74$ & $28 \pm 0.79$ \\
\hline C59 & Yulinli & $2,680 \pm 35.02$ & $3,800 \pm 31.62$ & $42.6 \pm 0.77$ & $40.5 \pm 0.91$ \\
\hline C60 & Yiqiaomili & $2,600 \pm 35.78$ & $5,590 \pm 50.4$ & $38 \pm 0.53$ & $35.3 \pm 0.74$ \\
\hline C61 & Jiaopanli & $2,544 \pm 43.57$ & $3,440 \pm 67.82$ & $46 \pm 0.8$ & $54.6 \pm 0.71$ \\
\hline C62 & Baisheng & $2,400 \pm 44.42$ & $6,460 \pm 50.99$ & $42.7 \pm 0.64$ & $42.63 \pm 0.67$ \\
\hline C63 & Haiken 4 & $2,360 \pm 51.81$ & $2,750 \pm 47.33$ & $24.49 \pm 0.76$ & $36 \pm 0.87$ \\
\hline C64 & Wuchali & $2,064 \pm 28.22$ & $4,980 \pm 20.98$ & $29.5 \pm 0.84$ & $10.77 \pm 0.41$ \\
\hline C65 & Haiken 14 & $960 \pm 28.6$ & $3,620 \pm 44.27$ & $22.18 \pm 0.82$ & $21.2 \pm 0.62$ \\
\hline
\end{tabular}

filament extends to half of its overall length, the anther has not yet achieved maturity. As the male flowers develop, the amount of mature pollen increases constantly in the anther. The amount of mature pollen reaches its maximum when the anther becomes fully mature. More pollen is needed in the crossbreeding of litchi. Theoretically, the fully mature anthers that are not dehiscent should be collected. In practice, because anthers develop nonsynchronously in the same tassel, it would be inefficient to collect fully mature anthers from every tassel. Thus, to ensure the maturity of the pollen and improve the collection efficiency of the pollen, anthers should be collected at the second and third development stages of male flowers every time. 
Presently, more than 500 accessions are preserved in the National Litchi Germplasm Repository located at the Institute of Fruit Tree Research, Guangdong Academy of Agricultural Sciences, Guangzhou, China, which was established in 1988 and is the largest litchi germplasm gene bank in the
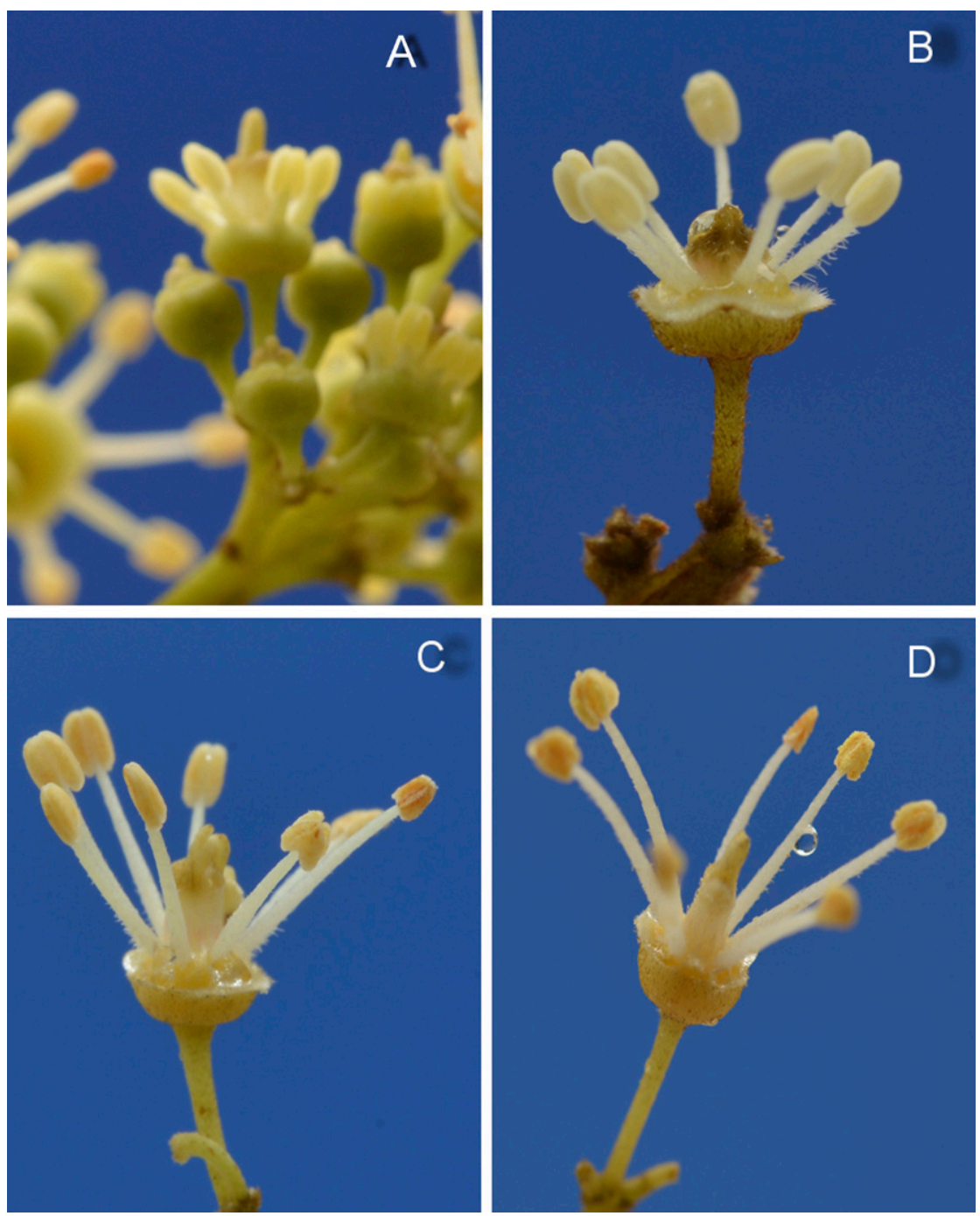

Fig. 1. (A) Litchi male flowers in the first stage, the anther extended just beyond the receptacle, and the filaments were not observed. (B) Litchi male flowers in the second stage, the filament is extended to half of its overall length. (C) Litchi male flowers in the third stage, the filament extends fully, but the anther does not dehisce. (D) Litchi male flowers in the last stage, the filament extends fully, and the anther dehisces.

world (Liu et al., 2015; Sun et al., 2010). A rich diversity exists among litchi cultivars. This is the first study of pollen diversity among 65 litchi cultivars. The average number of pollen grains per anther of the 65 tested cultivars ranged between 1555 and 7455. Significant differences were observed grown under subtropical conditions in São Paulo, Brazil, and the pollen grains per flower ranged between 23,000 and 74,000 (Campo Dall'Orto et al., 1985). On an average, Brazilian apple cultivars, such as Baronesa, Suprema, Imperatriz, Lisgala, Joaquina, Princesa, Fred Hough, Daiane, Catarina, Primícia, Duquesa and Condessa produce $16-20$ anthers, and a variable number of pollen grains per flower ranging between 53,000 and 103,700 were found. The variation in pollen grains per flower is mainly due to cultivar differences (Albuquerque et al., 2010). Each male litchi flower has 6-10 anthers (Wu, 1998). Thus, pollen grains per male flower ranged between 9330 and 59,640 for 65 litchi cultivars. Litchi panicles have a larger quantity of flowers, and the male flowers can produce large amounts of pollen grains during the flowering period.

Pollen collection is difficult but it is important. Adjusting the amount of pollen in artificial cross breeding in litchi based on the viability of the litchi variety conserves the amount of pollen. The differences among cultivars and between years in pollen viability, intended as flower quality, might be attributed to both physiological and environmental factors, but a genetic influence might play a significant role (Mazzeo et al., 2014). The litchi cultivar Feizixiao had lower pollen viability than 'Baitangying' (Jiang et al., 2012). The differences among cultivars observed in the present study are certainly indicative of the intervarietal variability, which is also detected in other fruit species, such as apples, peaches, plums, grapes, almonds, and sour and sweet cherries (Godini, 1981; Oberle and Goertzen, 1952; Szabó et al., 1996). Some authors have also reported significant differences in both the viability of clones of 'Arbequina' (Rovira and Tous, 2002) and the germination of clones of 'Leccino' (Bartolini and Guerriero, 1995). The differences among cultivars in litchi pollen viability attributed to genetic influence might play a significant role too. The weather

Table 3. Classification of the pollen quantity and germination rate of different litchi cultivars.

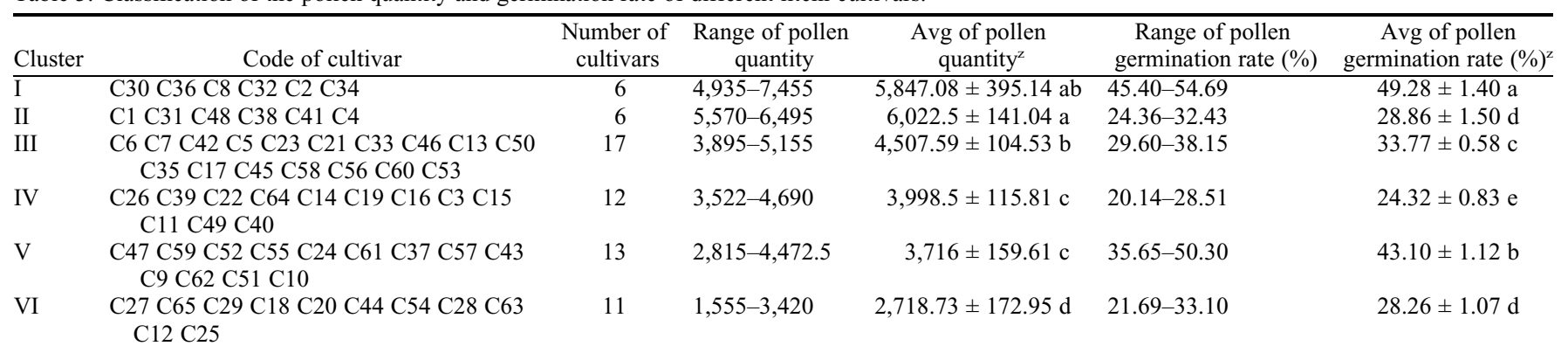

${ }^{\mathrm{z}}$ Different letters indicate significant difference among stages at $P=0.05$ (Tukey's test). 
affected the pollen germination rates in flowers bloom. The pollen germination rates of four olive cultivars had a significant difference between the 'on' and 'off' years (Gabriela et al., 2013). The litchi pollen quantity and viability had difference between 2009 and 2010. The differences among cultivars and between years in pollen quantity and viability mainly attributed to physiological and environmental factors. The rainy season in 2010 was started nearly a month later than 2009 in Guangzhou. The average temperature in 2010 was $19.75^{\circ} \mathrm{C}$, higher than $17.45{ }^{\circ} \mathrm{C}$ in 2009 . The pollen quantity and viability of most early-maturing varieties in 2010 were higher than 2009. The pollen quantity and viability of most late-maturing varieties in 2010 were lower than 2009. The results indicated that the pollen quantity and viability can be improved on warm and sunny days.

The hierarchical cluster based on pollen quantity and pollen germination rate showed that the 65 litchi cultivars could be classified into six clusters (Table 3 ). Six cultivars in the first cluster had high pollen quantity (4935$7455)$ and a high germination rate $(45.40 \%$ to $54.69 \%)$. The cultivars in the second cluster had high pollen quantity (5570-6495) but a low germination rate $(24.36 \%$ to $32.43 \%)$. The cultivars in the fifth cluster had a high pollen germination rate (35.65\% to $50.30 \%)$, but low pollen quantity (2815-4472.5). A clear relationship between pollen quantity and pollen germination rate in litchi was not found. Moreover, none of the cultivars had a pollen quantity and germination rate of 0 . Litchi cultivars producing the greatest amount of viable and compatible pollen grains per anther may be preferred when choosing potential pollinizers. This study highlighted some remarkable differences among cultivars in terms of both viability and number of pollen grains. Floral overlap is common among litchi cultivars and is the main mechanism that facilitates cross pollination. To optimize pollination, it is necessary to plant both early- and late-blooming pollinizers so that the main variety can bloom in between these time periods (Delaplane and Mayer, 2000). However, high pollination also depends on the occurrence of honeybees (three hives per hectare) (Guerrero Prieto et al., 2006). This study provides a theoretical basis for the collection of pollen, the quantity of pollen, and the amount of pollen used in pollination.

\section{Conclusions}

Differences among litchi cultivars were detected in this study, either for pollen production or viability. Concerning the pollination efficiency of litchi cultivars in general, the pollen quantity and germination rate could be important characteristics considered as indicative parameters of pollen development and functionality. Most litchi cultivars can produce large amounts of viable and compatible pollen grains during the flowering period. Pollen production may not be the main restriction for unsuccessful pollination of litchi. Flowering asynchrony, raining in bloom, and a lack of pollinators may be the main restrictions for unsuccessful pollination of litchi.

\section{Literature Cited}

Aizen, M.A. and L.D. Harder. 2007. Expanding the limits of the pollen-limitation concept: Effects of pollen quantity and quality. Ecology 88: 271-281.

Albuquerque, C.L., F. Denardi, A. Cd, M. Dantas, and R.O. Nodari. 2010. Número deanteras por flor, grãos de pólen por anterae capacidade germinativa do pólende diferentes cultivares de macieiras. Rev. Bras. Frutic. 32:1255-1260.

Bartolini, S. and R. Guerriero. 1995. Selfcompatibility in several clones of oil olive cv. Leccino. Adv. Hort. Sci. 9:71-74.

Campo Dall'Orto, F.A., W. Barbosa, M. Ojima, D.E. Ferraz, and S.A. Campos. 1985. Análisedo pólen em dezoito cultivares de macieira. Bragantia 41:421-427.

Dafni, A.D., P.G. Kevan, and B.C. Husband. 2005. Practical pollination biology. Enviro quest, Canada.

Delaplane, K.S. and D.F. Mayer. 2000. Crop pollination by bees. CABI, New York, NY.

Godini, A. 1981. Counting pollen grains of some almond varieties by means of an haemocytometer. Riv. Ortoflorofrutt. It. 65:173-178.

Gabriela, V.S., P. Slavko, G.B. Smiljana, and P. Milan. 2013. The effect of temperature and genotype on pollen performance in olive (Olea europaea L.). Sci. Hort. 156:38-46.

Guerrero Prieto, V.M., A. Romo Chacón, J.A. Orozco Avitia, D.I. Berlanga Reyes, A.A. Gardea Béjar, and R.Á. Parra Quezada. 2006. Polinización en manzanos red delicious y golden delicious. Rev. Fitotec. Mex. 29:41-45.

Jiang, S.Y., H.Y. Xu, H.C. Wang, G.B. Hu, H.B. Chen, and X.M. Huang. 2012. A comparison of the costs of flowering in 'Feizixiao' and 'Baitangying' litchi. Sci. Hort. 148:118-125.

Liu, W., Z.D. Xiao, X.L. Bao, X.Y. Yang, J. Fang, and X. Xiang. 2015. Identifying litchi (Litchi chinensis Sonn.) cultivars and their genetic relationships using single nucleotide polymorphism (SNP) markers. PLoS One 10(8): e0135390.

Mazzeo, A., M. Palasciano, A. Gallotta, S. Camposeo, A. Pacifico, and G. Ferrara. 2014. Amount and quality of pollen grains in four olive (Olea europaea L.) cultivars as affected by 'on' and 'off' years. Sci. Hort. 170:89-93.

Menzel, C. 2001. The physiology of growth and cropping in lychee. Acta Hort. 558:175-184.

Menzel, C.M. and G.K. Waite. 2005. Litchi and Longan. Botany, production and uses. CABI, Wallingford, UK.

Oberle, G.D. and K.L. Goertzen. 1952. A method for evaluating pollen production of fruit varieties. Proc. Amer. Soc. Hort. Sci. 59:263-265.

Ou, L.X., Q.M. Sun, J.Z. Chen, X.R. Wang, X. Xiang, and C.H. Cai. 2010. The report of litchi pollen characteristics research. The Ninth Guangdong Horticultural Society General Meeting and Academic Seminar, Guangzhou, China. p. 112-116.

Rovira, M. and J. Tous. 2002. Pollen viability in several 'Arbequina' olive oil clones. Acta Hort. 586:197-200.

Stern, R.A. and S. Gazit. 1996. Lychee pollen by the honeybee. J. Amer. Soc. Hort. Sci. 120: $152-157$.

Stern, R.A. and S. Gazit. 1998. Pollen viability in lychee. J. Amer. Soc. Hort. Sci. 123:41-46.

Sun, Q.M., L.X. Ou, X. Xiang, J.Z. Chen, Y.P. Qiu, Z.Q. Li, and C.H. Cai. 2010. Progress in breeding for litchi (Litchi chinensis Sonn.). J. Fruit Sci. 27:790-796. (in Chinese).

Szabó, Z., E. Felhösné Váczi, E. Csoma, Z. Kun, and J. Nyéki. 1996. Morphological char-acteristics of the flowers of some sour and sweet cherry varieties. Acta Hort. 410:127-131.

Wu, S.X. 1998. China fruit trees, litchi. 5th ed. China Forestry Publishing House, Beijing, China (in Chinese).

Xiang, X., L.X. Ou, Y.P. Qiu, Z.Q. Li, and J.Z. Chen. 2000. The affection of the chemical factors in litchi pollen vitality. Guangdong Agr. Sci. 6:29-32. (in Chinese).

Xiang, X., L.X. Ou, Y.P. Qiu, P.Y. Yuan, and J.Z. Chen. 2001. Embryo abortion and pollen parent effects in 'Nuo Mi Ci'and 'Gui Wei' litchi. Acta Hort. 558:257-260.

Xiang, X., Z.W. Zhang, B.Q. Wang, P.Y. Yuan, and Y.P. Qiu. 1994. The study of litchi pollen sterility and storage. Guangdong Agr. Sci. 4:25-27. (in Chinese).

Xu, D.Z., X.Y. Zhou, J.J. Zhuang, P.X. Mao, Z.S. Lin, and J.W. Wang. 1982. Measuring litchi pollen viability and germination condition. Fujian Fruits 2:28-32. (in Chinese).

Yu, C.C., T.S. Lin, and J.C. Chang. 2015. Pollen effects on fruit set, seed weight, and shriveling of '73-S-20' litchi-with special reference to artificial induction of parthenocarpy. HortScience 50:369-373. 